\title{
Meccanismo focale del terremoto del Friuli del 6 Maggio 1976
}

\author{
(The focal mechanism of the Friuli (Italy) earthquake \\ occurred on May 6th, 1976)
}

\author{
R. Console*
}

Ricevuto il 20 Ottobre 197 ;

\begin{abstract}
Riassusto. - Con metodi classici è stata determinata la soluzione per il mecenismo focale della scossa principale avvenuta in Friuli il 6 Magrgio 1976.

1 dati a nostra disposizione hanno consentito di fissare in maniera soddisfacente la posizione dei piani nodali che è risultata indieativa di un meccanismo di tipo dip-slip. Il piano di scorrimento è inclinato di circa 1 i) verso $\mathrm{N} N \mathrm{~W}$.
\end{abstract}

Sumary. - TBy classical methods the focal mechanism solution for the Friuli May 6th 1976 earthquake has been determined.

The data in our possess allowed us to fix satisfactorialy the nodal plane position, which indicated a dip-slip mechanism. The fault plane is dipping about $15^{\circ}$ towards $\mathrm{NNW}$.

\section{1. - INTRODUZIONE}

Il periorlo sismico manifestatosi nel Friuli durante il 1976 ha aruto inizio il 6 Maggrio alle ore 19.59 con la scossa premonitoria di magniturlo $M_{\imath}=4.5$ (RMP), seguita subito dalla scossa principale (lelle $20 \mathrm{~h} 00 \mathrm{~m} 11 \mathrm{~s}$ la cui magniturlo ì stata valutata pari a $1 K_{\iota}=6.2$

* Istifuto Nazionale di Geofisica, Roma. Osservatorio Geofisico Centrale, Ionteporzio Catone. 
(RAP). L'epicentro clella scossa principale è stato localizzato dall'I.N.G. nel punto di coordinate

$$
\begin{aligned}
& \varphi=46.253^{\circ} \mathrm{N} \\
& \lambda=13.240^{\circ} \mathrm{E}
\end{aligned}
$$

mentre, per quanto riguarda la profondità dell'ipocentro, la scarsità lelle stazioni vicine non ne ha consentito una valutazione abbastanza accurata. Tale profondità, confermata anche dall'anclamento delle isosiste, e dalle posizioni delle repliche, dovebbe aggirarsi intorno ai $10 \div 15 \mathrm{~km}$.

In questo lavoro ai si ̀̀ proposti di eseguire la determinazione del meccanismo focale della scossa principale del 6 Magcrio, indivicluando la direzione e l'inclinazione del piano lungo il quale si è verificata la frattura considerata causa del terremoto.

Come i noto, i meccanismi focali dei terremoti vengono studiati in base ai rersi dei primi impulsi registrati nelle stazioni sismiche. $\Lambda$ questo scopo occorrono registrazioni di buona qualità, nelle quali il senso del movimento dell'onda P possa essere inclividuata senza incertezza.

Inoltre ò opportuno che le stazioni utilizzate nel calcolo siano distribuite quanto più miformemente possibile in azimut ed in distanza rispetto all'epicentro. Queste condizioni famo si che i meccanismi focali possano essere studiati con profitto solo per le scosse di notevole magnitudo.

La scossa del 6 Maggio di magnitudo 6.2 può consiclerarsi idonea all'applicazione di questo metodo, sebbene un'altra circostanza abbia reso incerta l'analisi dei sismogrammi per le stazioni vicine a breve periorlo. Infatti, essenclo stata precerluta dalla premonitrice di magnituclo 4.5, essa ha dato luogo a registrazioni il cui inizio era noterolmente confuso nel corpo dell'evento precerlente.

\section{2. - IL METODO.}

Pel il calcolo della soluzione del meccanismo focale si è adoperato un programma tratto da quello pubblicato da Wickens e Hodgson $\left(^{3}\right)$ nel 1967. Tale programma assume un modello di sorgente a doppia coppia pervenendo ai tre parametri caratterizzanti l'orientamento nello spazio dei relativi piani nodali in modo che essi soddisfino nel miglior modo i risultati sperimentali. 
Per prima cosa vengono determinati l'azimut e la distanza delle stazioni utilizzate nel calcolo, rispetto all'epicentro. Quindi, in base alla teoria dei raggi sismici ed alle tarole dei tempi di tragitto di Jeffreys e Bullen, si determina l'angolo $i$ col quale le onde sismiche hanno lasciato l'ipocentro del sisma, per le singole stazioni.

Il procedimento seguito consiste nel determinare una certa funzione di merito, ottenuta dalla somma dei singoli contributi che le stazioni forniscono in base ad un peso che dipende dalla loro posizione relativa ai piani nodali. Questa funzione ha il suo massimo quando la posizione dei piani consente di separare nel modo ottimale $\mathrm{i}$

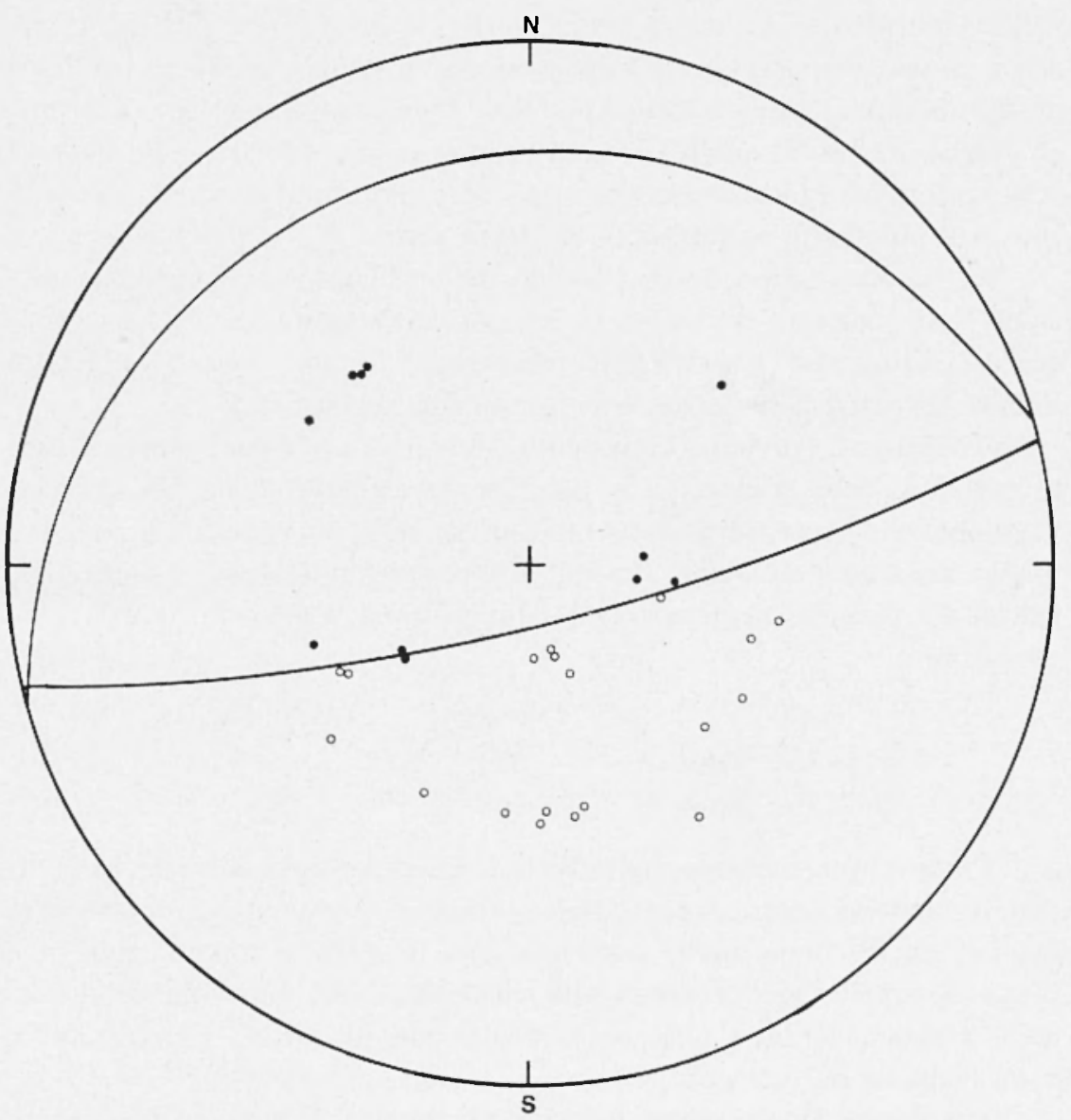

Fig. 1 - Rappresentazione su proiezione stereografiea di Wulff del meccanismo focale della scossa del 6 Maggio 1976 , II $=20 \mathrm{~h} 00 \mathrm{mlls}$. 
gruppi di stazioni che hanno registrato o una dilatazione o una compressione. La posizione dei piani che rerifica tale situazione viene considerata soluzione del problema.

Per la rappresentazione grafica della soluzione si è scelta la proiezione stereografica di Wulff della semisfera focale inferiore. Su questa proiezione le compressioni e le dilatazioni vengono separate da due linee che rappresentano cerchi massimi perpendicolari fra loro.

\section{3. - Applicazione al terremoto del 6 Maggio.}

Per il calcolo dei parametri del meccanismo focale sono state utilizzate tutte le informazioni riguardanti le polarità dei primi arrivi a nostra disposizione, senza discriminazione tra quelle provenienti da sismografi a breve e lungo periodo. I parametri ipocentrali forniti al programma sono quelli citati in premessa. La velocità delle onde $P$ alla profondità ipocentrale, ricarata dal programma stesso in accordo con le dromocrone fornitegli, è risultata pari a $V_{m}-6.2 \mathrm{~km} / \mathrm{sec}$.

In tabella 1 riportiamo l'elenco delle stazioni adoperate nel calcolo. Nelle colonne adiacenti si ha rispettivamente: la distanza epicentrale, l'azimut rispetto all'epicentro, l'angolo preso dal raggio sismico rispetto alla verticale e la polarità registrata.

Nella fig. 1 troviamo rappresentate con la proiezione stereografica le posizioni delle stazioni e la polarità da esse registrata con la convenzione che il cerchietto bianco corrisponde ad una dilatazione e quello nero ad una compressione. I semicerchi neri rappresentano le tracce dei piani nodali ricavati dal programma, i cui poli hanno come coordinate:

$\begin{array}{lcc} & \text { Direzione } & \text { Inclinazione } \\ \text { polo del piano } \Lambda & 344^{\circ} & 14.5^{\circ} \\ \text { polo del piano } \mathrm{C} & 168^{\circ} & 75.5^{\circ}\end{array}$

Come si può notare, quasi tutti i dati sperimentali sono coerenti con il modello trovato per cui la soluzione è da ritenersi piuttosto attendibile. Si deve anche osservare che per fissare l'inclinazione del piano A (quello più prossimo alla verticale) sono determinanti i dati delle stazioni lontane che, nella proiezione di Wulf, riguardano la zona centrale del disegno.

Per fissare l'inclinazione rlel piano $\mathrm{C}$, invece, le stazioni fondamentali sono quelle vicine, la cui distribuzione è risultata inadeguata al caso in esame. 
Tabella 1

\begin{tabular}{|c|c|c|c|c|c|}
\hline \multicolumn{2}{|c|}{ Sigla-Stazioni } & \multirow{2}{*}{$\begin{array}{c}\text { Distanze pi- } \\
\text { centrali } \Delta^{0} \\
0.6\end{array}$} & \multirow{2}{*}{$\begin{array}{c}a^{o} \\
145.9\end{array}$} & \multirow{2}{*}{$\begin{array}{c}\begin{array}{c}\text { angolo } \\
i^{\circ}\end{array} \\
60.2\end{array}$} & \multirow{2}{*}{$\begin{array}{c}\text { Polarità S } \\
-\end{array}$} \\
\hline TRI & Trieste & & & & \\
\hline P'AD & Padova & 1.3 & 228.7 & 53.0 & - \\
\hline VKA & Vienna & 2.9 & 45.7 & 52.5 & + \\
\hline$A Q U$ & Aquila & 4.0 & 178.3 & 52.5 & - \\
\hline BII & Buchlerhoehe & 4.2 & 307.3 & 52.4 & + \\
\hline $\mathrm{RMIP}$ & Roma MI.P'. & 4.5 & 185.2 & 52.3 & - \\
\hline $\mathrm{NPI}_{4}$ & Napoli Ves. & 5.5 & 170.8 & 51.9 & - \\
\hline $\mathrm{BHO}$ & Beograt & 5.3 & 103.2 & 51.9 & - \\
\hline STB & Steinbach & 6.1 & 317.9 & 51.7 & + \\
\hline $\mathrm{BNS}$ & Bensberg & 6.2 & 321.7 & 51.7 & +- \\
\hline $\mathrm{BEG}$ & Berghein & 6.4 & 319.6 & 51.6 & + \\
\hline \multirow[t]{2}{*}{ SKO } & Skopje & 7.3 & 123.0 & 51.2 & - \\
\hline & Cagrliari & 7.7 & 204.8 & 51.1 & - \\
\hline MES & Messina Univ. & 8.2 & 167.1 & 50.8 & 一 \\
\hline A'TU & Atene Iniv. & 11.3 & 133.1 & 49.0 & - \\
\hline IST & Istanbul & 12.5 & 108.9 & 47.9 & - \\
\hline $\mathrm{T}(\mathrm{OI} /$ & 'Toledo & 14.1 & 249.4 & 47.0 & + \\
\hline $\mathrm{M} \Lambda \mathrm{I}$ & Malaga & 16.3 & 240.4 & 44.8 & - \\
\hline N KM & Nakhla & 17.7 & 239.1 & 43.7 & - \\
\hline BME & Barrage & 21.8 & 232.3 & 33.7 & + \\
\hline $\mathrm{TAB}$ & Tabriz & 25.7 & 96.6 & 31.1 & + \\
\hline SHI & Shiraz & 34.7 & 104.6 & 28.5 & - \\
\hline NDI & New Delhi & 52.3 & 86.1 & 24.6 & + \\
\hline$P 00$ & Poona & 56.6 & 98.0 & 23.3 & + \\
\hline I, IV I & Lwiro & 50.2 & 159.6 & 25.1 & - \\
\hline BUI, & Bulawayo & 67.5 & 164.4 & 20.4 & - \\
\hline WIN & Windhoek & 68.6 & 176.2 & 20.1 & - \\
\hline PRE & Pretoria & 72.9 & 165.9 & 18.9 & - \\
\hline
\end{tabular}

Infatti, come si ̀̀ accemnato nella premessa, molte registrazioni erano già saturate dalle onde provenienti dal terremoto arrenuto circa un minuto prima.

DISCUSSIONE DEI RISUTTATI.

Come è noto, la soluzione del problema del meccanismo focale di un terremoto presenta, dal punto di vista fisico, l'ambiguità nel- 
l'individuazione del vero piano di faglia tra i due piani nodali. Talvolta tale ambiguità può essere eliminata con considerazioni geologiche.

Nel nostro caso la soluzione indica chiaramente un mecanismo di tipo rip-slip, nel quale uno dei piani è molto più inclinato dell'altro rispetto alla verticale. La scelta del piano di faglia tra i due piani complementari, va fatta chiaramente in accordo con il fatto che il blocco settentrionale (le $A l p i)$ presenti un solleramento rispetto a quello meridionale (la Valle Padana).

Ciò risulta possibile solo prendendo come piano di scorrimento il secondo piano nodale. Si tratta, quindi, di un movimento in cui le Alpi sovrascorrono verso Suc o, viceversa, di un movimento in cui l'Italia sottoscorre verso Nord.

I risultati qui esposti son in eccellente accordo con quanto già pubblicato da G. Mrueller $\left.{ }^{1}\right)$, il quale li ha ricavati dallo studio delle registrazioni a lungo periodo della rete WWSSN.

\section{BIBLIOGRAFIA}

(1) Munduer G., 1976. - Fault plane solution of the earlhqualie in Northern Ilaly, o May 19\%6, and implications for the lectonies of the Eastern Alps. "Journal of Geophysies".

(2) Publication. - The mechanics of faulling, wilh special reference to the faull-plane work. "Dominion Observatory", Ottawa, XX, 2.

${ }^{3}$ ) Wickess A. J., IIODGoss J. H., 1967. - Compuler revalualion of earlhquale mechanism solutions 1922-1962. "Dominion Observatory", XXIII, I. 